\title{
Practical interval type-2 fuzzy self-tuning of PID controller to servo permanent magnet synchronous motor
}

\author{
M. A. Abdel Ghany ${ }^{1 *}$, M. E. Bahgat ${ }^{2}$, W. M. Refaey ${ }^{2}$ and Soliman Sharaf ${ }^{2}$
}

*Correspondence:
mghany1988@hotmail.com
${ }^{1}$ Faculty of Engineering,
October 6 University, Cairo,
Egypt
Full list of author information
is available at the end of the
article

*Correspondence: mghany1988@hotmail.com Faculty of Engineering, Egypt article

\begin{abstract}
This paper implements a practical interval type-2 fuzzy self-tuning (IT2FST) of optimal PID (OPID) controller to servo permanent magnet synchronous motor (SPMSM). The proposed method IT2FST updates the OPID controller gains in an online manner to drive the SPMSM with better speed response during variable load and parameter uncertainty occurrence. In this work, the industrial SPMSM system comprises threephase PMSM with internal break, drive and mechanical parts. Due to the incomplete real information of the SPMSM, nonlinear least square algorithm has been utilized for its model identification. A comparative analysis in a real time of the SPMSM with an OPID, type-1 fuzzy self-tuning and IT2FST for OPID controllers under the influence of parameter uncertainties and external load disturbances has been carried out. The real-time practical implementation results illustrated that the proposed IT2FST of OPID controller gives a simple opportunity to enhance the speed performance of the SPMSM than the other controllers.
\end{abstract}

Keywords: Permanent magnet synchronous motor, Identification, Interval type-2 fuzzy self-tuning for PID control

\section{Introduction}

The permanent magnet synchronous motors (PMSMs) have many applications in industries due to their compact structure, high efficiency, high power density and hightorque-to-inertia ratio [1]. The PI/PID controller is unquestionably the most commonly used control algorithm in the process control of industrial servo motor system [2]. The main reason for this usage is its relatively simple structure, which can be easily understood and implemented in practice. In spite of its widespread use, there exists no generally accepted design method for the controller. PI and PID controllers have traditionally been tuned empirically, e.g., by the method described in Ziegler and Nichols [3]. This method has the great advantage of requiring very little information about the process. There is, however, a significant disadvantage because the method inherently gives very poor damping [3, 4]. The tuning of electric drive controller is a complex problem due to the many nonlinearities of the machines and power converter. Therefore, many tuning rules have been proposed for this type of controller. During the last three decades, one 
of the main focuses of research in control engineering has been devoted to providing automatic tuning of such controllers.

Self-tuning PI control technique based on neural network was executed in [5]. A comparative study of three popular evolutionary algorithms: genetic algorithms, particle swarm optimization and differential evolution, for optimal tuning of PI speed controller for PMSM drives is given in [6].

It was mostly observed that a combination of fuzzy self-tuning mechanism and PID controller is used to improve and enhance the transient system performance. In this case, the fuzzy self-tuning was used to tune the PID gains [7]. These studies only focus on the ordinary T1FST of PID controllers [7-9]. It has been shown in various works that the T1FST of PID controllers might not be able to fully handle the high levels of uncertainties associated with control applications. On the other hand, the interval type-2 fuzzy sets (IT2 FSs) might be able to handle such uncertainties to produce a better control performance $[10,11]$. The uncertainties are generally coming from the noise in the measurements and the parameter changing due to the environmental and operating conditions [13]. It has been shown that IT2-FPIDs achieve better control performances because of the additional degree of freedom provided by the footprint of uncertainty (FOU) in their antecedent MFs $[14,15]$. Nevertheless, a systematic design for type-2 fuzzy controllers is still a challenging problem due to the main difficulty in determining the IT2 FSs and rule base [16].

This paper proposes IT2FST of OPID, which was firstly proposed as a T1FST of OPID controller to PMSM servo system [17].

The simulation results and practical implementation for speed control of SPMSM system are based on MATLAB/Simulink toolbox. The benefit of this approach is examined via various practical studies performed on SPMSM system under load changing and influence of parameter uncertainties. The results clearly show that the proposed controller IT2FST OPID has better dynamic response, in the form of minimum overshoot and settling time in comparison with the OPID and T1FST of OPID controllers.

\section{Servo motor system and experimental setup}

The experimental platform is shown in Fig. 1. The experimental setup is designed for the theoretical and practical investigation studies of the proposed PMSM controllers. The experimental setup consists of six parts [17]:

1. SEW synchronous three-phase permanent magnet servo motor.

2. SEW MOVIDRIVE-B to drive the motor.

3. Mechanical load.

4. PC used to perform the control algorithms.

5. Interface adapter option USB11A.

6. A data acquisition card (DAQ) NI USB-6008.

The description of the main components of the SPMSM is illustrated in Fig. 2.

Motor It is a SEW synchronous three-phase permanent magnate servo motor with internal break. 


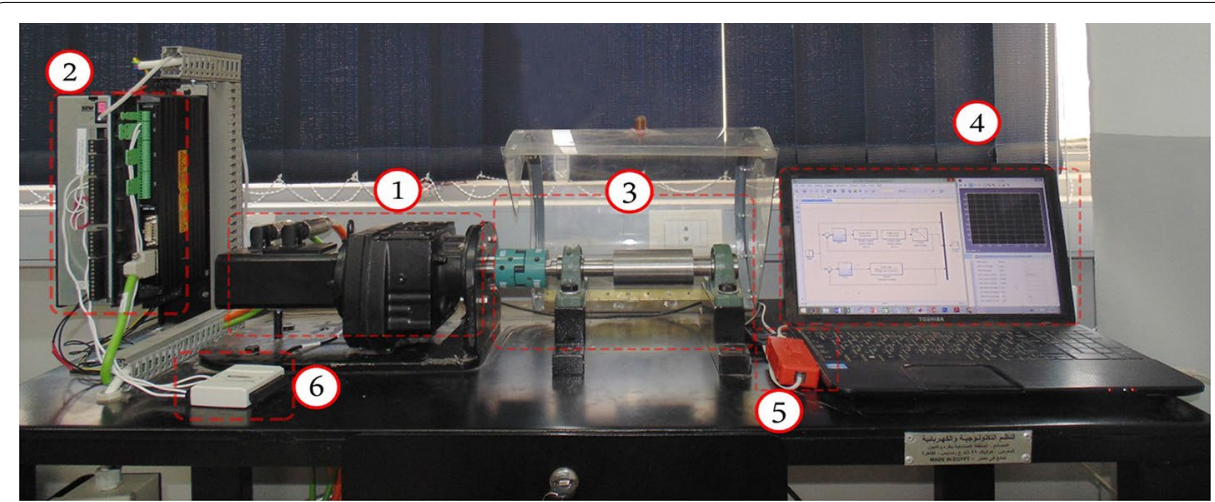

Fig. 1 Experimental setup of servo system

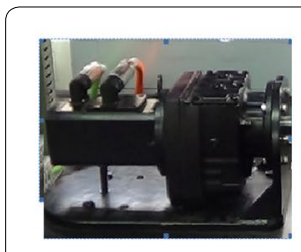

Motor

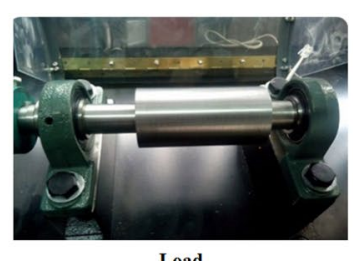

Load

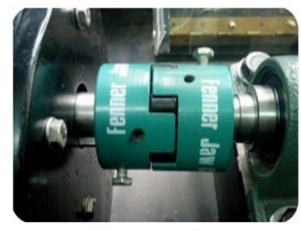

Coupling

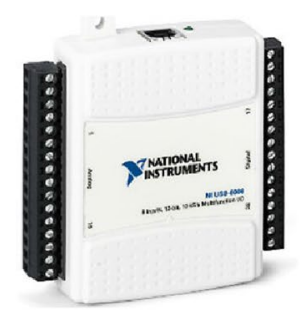

The Multifunctional DAQ NI USB-6008

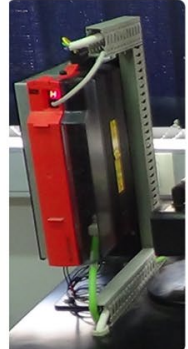

Drive

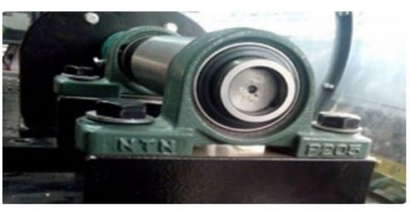

Bearing

Fig. 2 Servo permanent magnet synchronous motor and its main components

Load The mechanical load in this case is a variable to study variable inertia. Load is cylinder shaped.

Coupling It transfers motion from motor shaft to the load shaft.

Multifunctional data acquisition (DAQ) It represents the terminal parts for sending and receiving identification signals. It can take a continuous signal, and it can be sampled at evenly spaced interval to produce a series of discrete values that represent the original signal. In addition, DAQ is used to transmit the analog signal that represents the speed from the drive to the computer.

Drive It transfers motion from motor shaft to the load shaft, receives a command signal from a control system, amplifies the signal and transmits electric current to the motor in order to produce motion proportional to the command signal.

Bearing It reduces friction between moving parts and bearing with housing is used to fix the load. 


\section{Servo motor model identification}

In the experimental setup of PMSM system, the motor parameters were given in [17]. Precisely, the incomplete SPMSM real information for its components described in Fig. 2 made nonlinear least square algorithm (NLSA) is used for system model identification. System identification MATLAB toolbox is employed for this aim. Figure 3 shows the block diagram for servo system description.

\section{Model parameter identification}

The identification is to define a system among a specific category of models based upon input/output information which is equivalent to the system [17-19]. Generally, there are two major categories of identification: system identification and parameter identification. In the case of system identification, the equations of the system are usually unknown or vary during the process time. The target is to find a mathematical model describing the system, by giving appropriate inputs and observing the outputs. The system identification steps can be summarized as follows:

1. Data collection

2. Estimation of the parameters

3. Validation of the obtained model

4. Choice of the model structure

Based on the above, the identification input signal is an input voltage $v$. The output signal is represented by a voltage corresponding to the measured speed $\omega$ of motor. Figure 4 illustrates the construction of DAQ and its simulation in the Simulink toolbox [17]. The input/output signals using chirp function are illustrated in Fig. 5.

Different types of order of PMSM identification models are performed to get the best-fit model. The system identification is based on the nonlinear least square technique. The validation of the three-fit (tf3, tf6 and tf7) models by using different types of input signals (step, square and Sin) was performed to ensure the sustainability of the model response as shown in Fig. 6.

Based on the model validation of the experiential of the PMSM, the identified linear second order was the best selection as given [17].

The second order is selected as a model structure for describing the system according to wide range survey and many experimental trials (model tf3). The approximate transfer function of the proposed servo system may be written as follows:

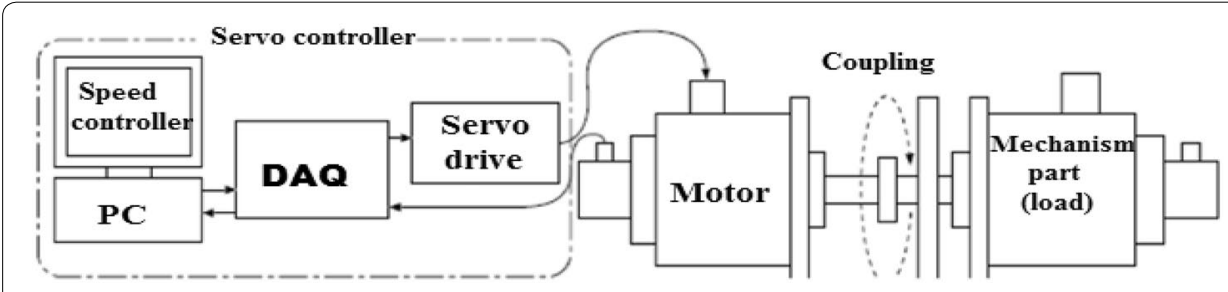

Fig. 3 Block diagram of description of servo system 

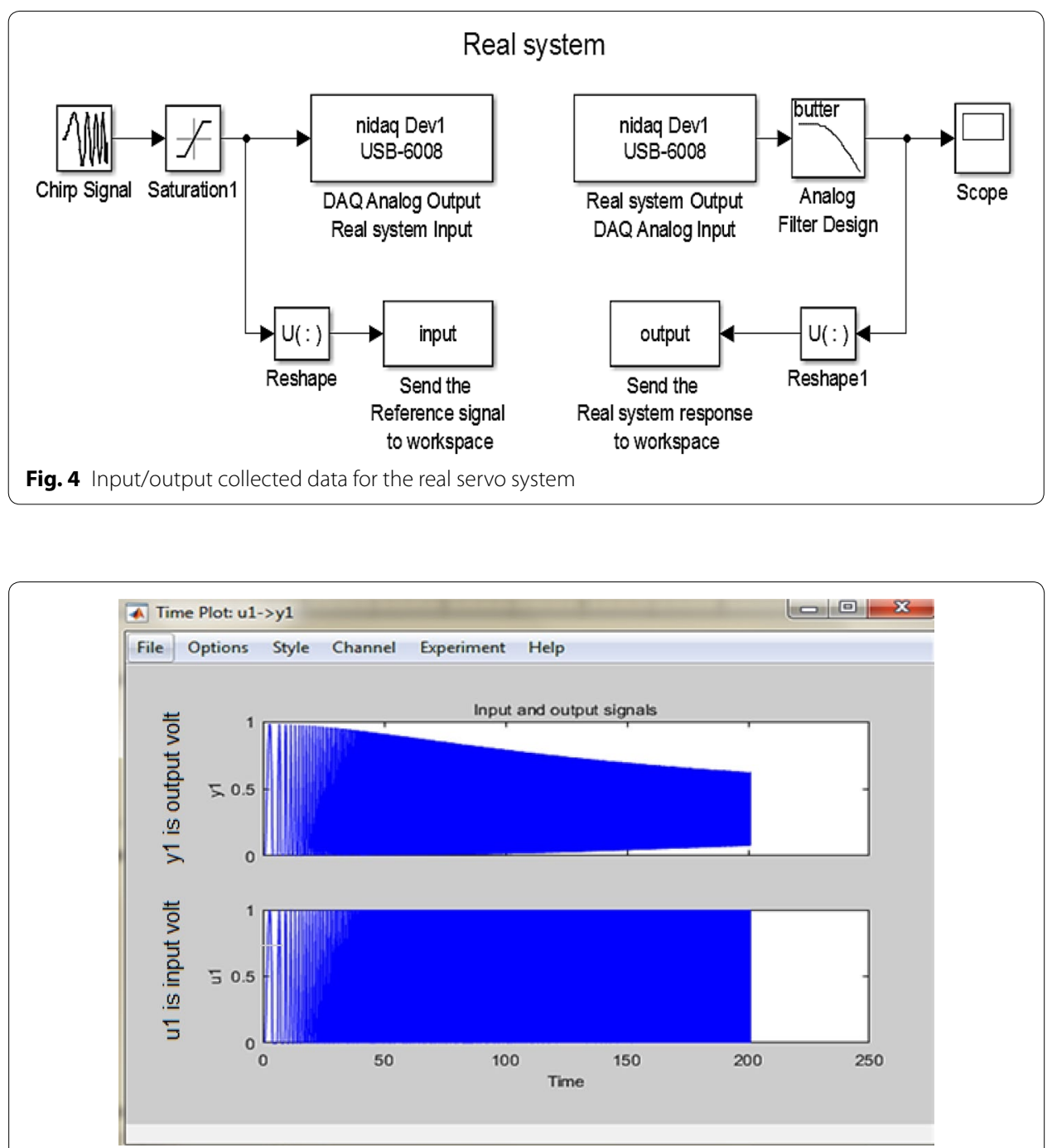

Fig. 5 Input/output collected data

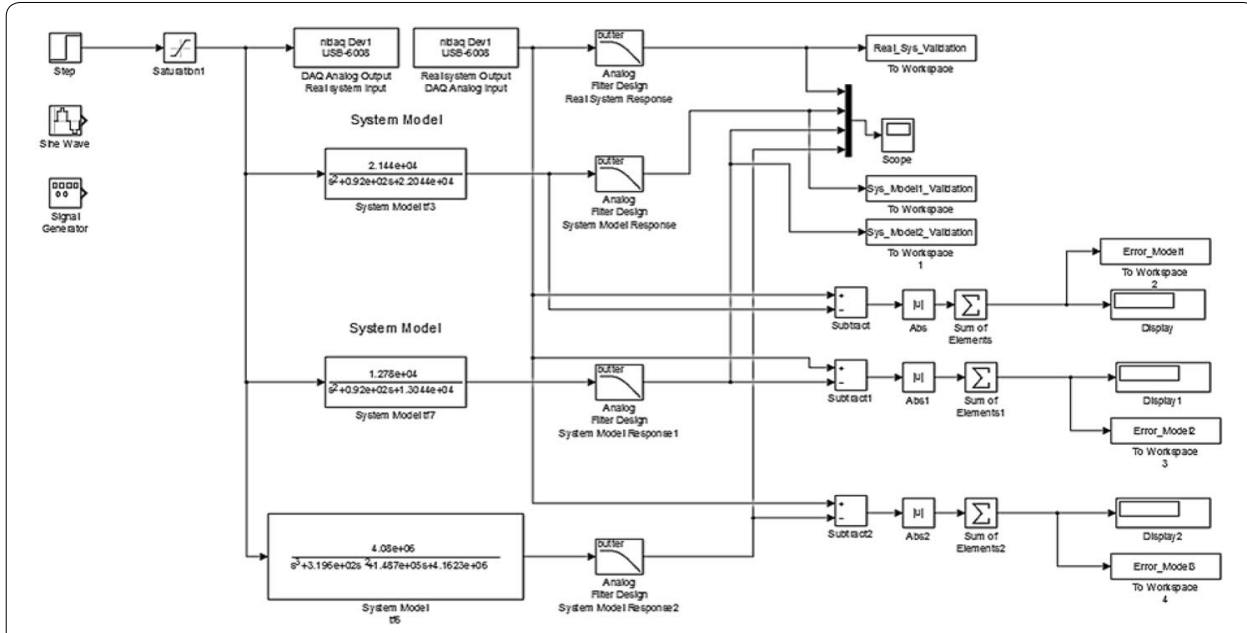

Fig. 6 Block diagram of system model validation 


$$
\frac{\omega(s)}{v(s)}=\frac{k}{a s^{2}+b s+c}
$$

where $\omega(s)$, rotor speed; $v(s)$, controlled voltage; and $k, a, b, c$, the transfer function parameters.

The transfer function representing the angular velocity $\omega$ of motor and the input motor voltage $v$ obtained from the system identification process could be expressed as [17]:

$$
\frac{\omega(s)}{v(s)}=\frac{2.144 \mathrm{e} 04}{s^{2}+0.92 \mathrm{e} 02 s+2.2044 \mathrm{e} 04}
$$

\section{Ant system optimization for PID controller and type-2 fuzzy system}

The equation of the PID transfer function is given in (3):

$$
K(s)=K_{\mathrm{p}}+\frac{K_{\mathrm{i}}}{s}+K_{\mathrm{d}} s
$$

where $K_{\mathrm{p}}, K_{\mathrm{i}}$ and $K_{\mathrm{d}}$ are the proportional, integral and differential gains, respectively. Ant colony optimization (ACO) is used to obtain optima PID gains. Using the ant colony optimization process to find the optimal parameters of the controller such that to minimize or maximize a given cost function of the closed loop system consisting of an ant based PID controller and an unknown plant [17] the effectiveness of the PID based ant colony was investigated by the following parameters variation test [20,21]. The performance criteria of the system were given in [17], and the optimal gains of the PID controllers were $K_{\mathrm{p}}=60, K_{\mathrm{i}}=20$ and $K_{\mathrm{d}}=3$.

\section{Type-2 fuzzy logic systems}

There are two different approaches for FLSs design: type-1 FLSs (T1FLSs) and type-2 FLSs (T2FLSs). The latter is proposed as an extension of the former. While designing a T1FLSs, expertise and knowledge are needed to decide both the MFs and fuzzy rules. The T1FLSs, whose MFs are type-1 fuzzy sets, are unable to directly handle rule uncertainties [12, 21]. To deal with this problem, the concept of type- 2 fuzzy sets was introduced by Zadeh as an extension of T1FLSs with the intention of being able to model the uncertainties that invariably exist in the rule base of the system [22].

\section{Type-2 fuzzy sets (T2 FSs)}

A T2 FS, denoted $\tilde{A}$, is characterized by a type-2 MF $\mu_{\tilde{A}}=(x, u)$, where $x \in X$ and $u \in J x \subseteq[0,1]$, i.e.,

$$
\tilde{A}=\left\{\left((x, u), \mu_{\tilde{A}}(x, u)\right) \mid \forall x \in X, \forall u \in J_{x} \subseteq[0,1]\right\}
$$

in which $0 \leq \mu_{A}(x, u) \leq 1 . \tilde{A}$ can also be expressed as

$$
\tilde{A}=\int_{x \in X} \int_{u \in J_{x}} \frac{\mu_{A}(x, u)}{(x, u)} J_{x} \subseteq[0,1]
$$

where $\iint$ denotes union over all admissible $x$ and $u$. For discrete universes of discourse, $\int$ is replaced by $\sum[12,21]$. 


\section{Interval type-2 fuzzy sets}

When all $\mu_{\tilde{A}}(x, u)=1, \tilde{A}$ is an interval T2 FS (IT2 FS). Although the third dimension of the general T2 FS is no longer needed because it conveys no new information about the IT2 FS, the IT2 FS can still be expressed as a special case of the general T2 FS in (5), as [22]:

$$
\begin{aligned}
& \tilde{A}=\int_{x \in X} \int_{u \in J_{x}} \frac{1}{(x, u)} J_{x} \subseteq[0,1] \\
& \tilde{A}=\int_{x \in D_{x}} \int_{u \in J_{x} \subseteq[0,1]} \frac{1}{(x, u)}=\int_{\in D_{x}}\left[\int_{u \in J_{x} \subseteq[0,1]} \frac{1}{u}\right] / x
\end{aligned}
$$

where $x$, called the primary variable, has domain $D_{\tilde{X}}: u \in[0,1]$, called the secondary variable, has domain $J_{x} \subseteq[0,1]$ at each $x \in D_{\tilde{X}}$; $J_{x}$ is also called the primary membership of $x$ and the amplitude of $\mu_{\tilde{x}}(x, u)$, called a secondary grade of $\tilde{A}$, equals 1 for $\forall x \in D_{\tilde{X}}$ and for $\forall u \in J_{x} \subseteq[0,1]$.

The upper membership function (UMF) and lower membership function (LMF) of $\tilde{A}$ are two T1 membership functions that bound the footprint of uncertainty (FOU) as shown in Fig. 7. The UMF of $\tilde{A}$ is the upper bound of the FOU $(\tilde{A})$ and denoted as $\bar{\mu}_{\tilde{x}}(x) \forall x \in X$, and the LMF is the lower bound of the FOU $(\tilde{A})$ and denoted as $\underline{\mu}_{\tilde{x}}(x) \forall x \in X$. The UMF and LMF can be characterized as follows [23, 24]:

$$
\begin{aligned}
& \bar{\mu}_{\tilde{x}}(x)=\overline{\operatorname{FOU}(\tilde{A})} \forall x \in X \\
& \underline{\mu}_{\tilde{x}}(x)=\underline{\operatorname{FOU}(\tilde{A})} \forall x \in X
\end{aligned}
$$

The computations of fuzzification and inference for IT2-FLC were given and discussed in [12, 22-24]. For this operation, type reduction to convert IT2-FLC into a T1-FLC is performed [23, 24]. There are several methods of type reduction. In this paper, the "center-of-sets" type reduction is used. The calculations of this method were done and given in [23]. In addition, the defuzzification method is determined to convert typereduced set to crisp output of an IT2-FLS [23, 24].

\section{Online interval type-2 fuzzy self-tuning for the OPID controller}

Figure 8 shows the block diagram of an IT2F-PID controller for SPMSM. For the system under study, the universe of discourse for both $e(t)$ and $\Delta e(t)$ for $K_{\mathrm{p} 2}, K_{\mathrm{i} 2}$ and $K_{\mathrm{d} 2}$

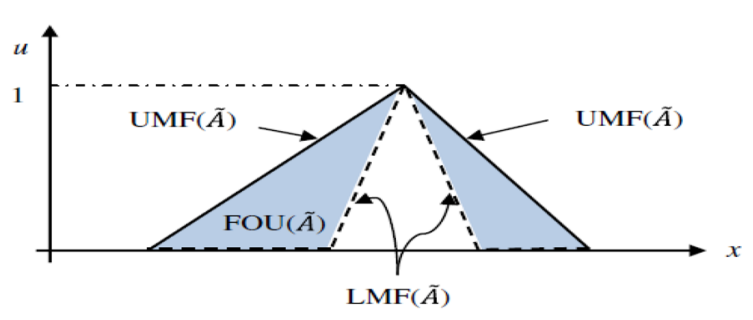

Fig. 7 FOU, UMF and LMF for an IT2FS $\tilde{A}$ 

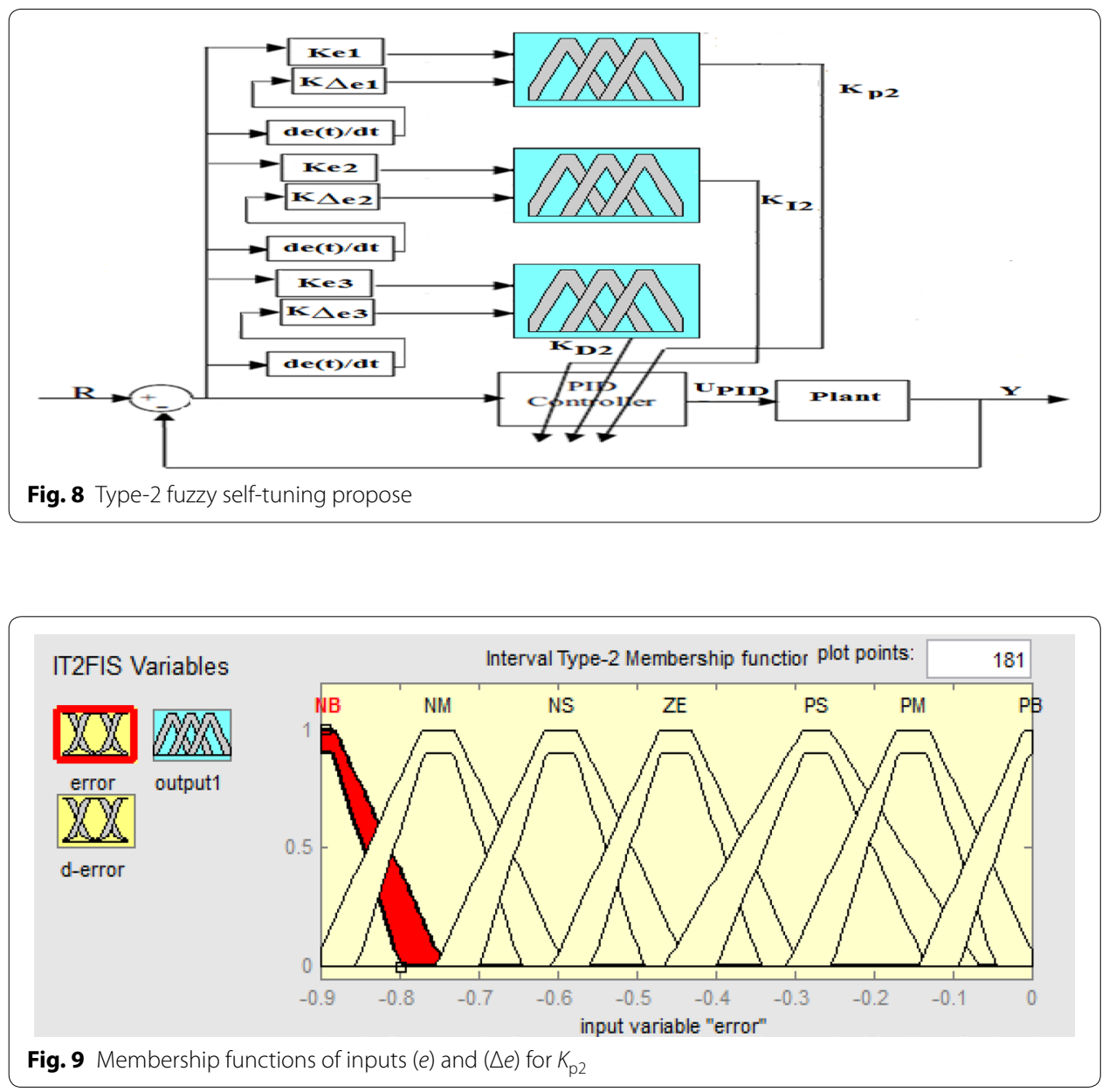

is normalized with $[-0.9,0],[-0.01,0]$ and $[-0.1,0]$, respectively, while the universe of discourse for each $K_{\mathrm{p} 2}, K_{\mathrm{i} 2}$ and $K_{\mathrm{d} 2}$ is normalized from [0, 5.5], [0, 10] and [0, 0.4], respectively. The linguistic labels are (negative big, negative medium, negative small, zero, positive small, positive medium, positive big\}, and the linguistic labels of the outputs are \{zero, medium small, small, medium, big, medium big, very big\}. The IT2 of membership function for $e(t)$ and $\Delta e(t)$ and for the output $K_{\mathrm{p} 2}$ is shown in Figs. 9 and 10, respectively. The membership functions for $e(\mathrm{t})$ and $\Delta e(t)$ and for $K_{\mathrm{i} 2}$ and $K_{\mathrm{d} 2}$ are similar to Figs. 9 and 10, respectively, but with different universes of discourse values.

The control rules used for T1FST of OPID controller for determining the output gains from fuzzy controller were given $[7,17]$.

This general equation of the PID can be written as:

$$
U=K_{\mathrm{p}}+K_{\mathrm{i}} \int e \mathrm{~d} t+K_{\mathrm{d}} \frac{\mathrm{d} e(t)}{\mathrm{d} t}
$$

This equation of the PID after fuzzy effect can be written as: 


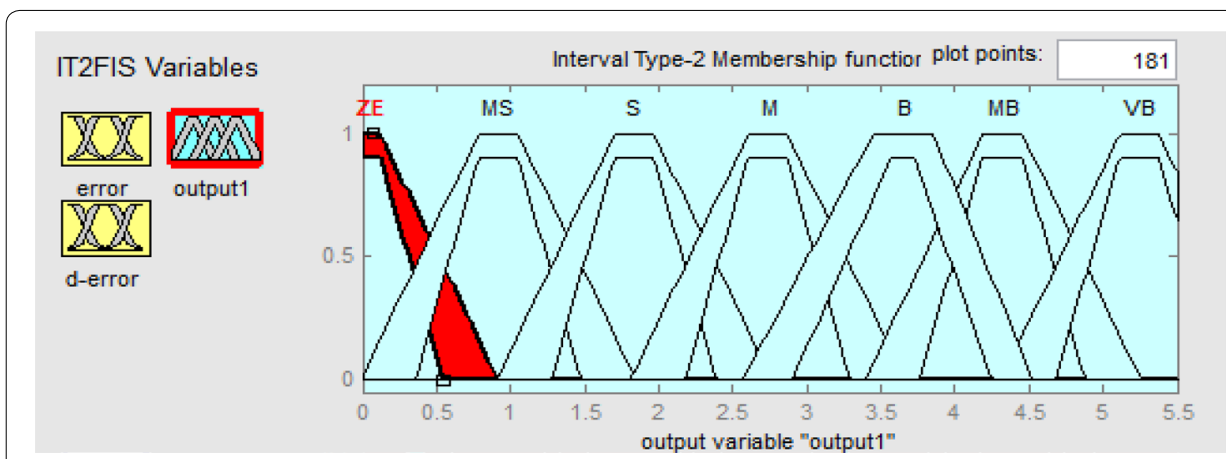

Fig. 10 Membership functions of outputs $K_{p 2}$

$$
U=K_{\mathrm{p} 3}+K_{\mathrm{i} 3} \int e \mathrm{~d} t+K_{\mathrm{d} 3} \frac{\mathrm{d} e(t)}{\mathrm{d} t}
$$

where

$$
K_{\mathrm{p} 3}=K_{\mathrm{p}} * K_{\mathrm{p} 2}, \quad K_{\mathrm{i} 3}=K_{\mathrm{i}} * K_{\mathrm{i} 2}, \quad K_{\mathrm{d} 3}=K_{\mathrm{d}} * K_{\mathrm{d} 2}
$$

$K_{\mathrm{p} 2}, K_{\mathrm{i} 2}$ and $K_{\mathrm{d} 2}$ are the output gains from fuzzy controller of IT2FST, where $K_{\mathrm{ei}}$ error input normalizing gain, $i=1,2,3 ; K_{\Delta \mathrm{e} i}, \Delta$ error input normalizing gain, $i=1,2,3$.

\section{Simulation and practical results}

Combination between MATLAB/Simulink toolbox and multifunctional DAQ with synchronous three-phase servo motor permanent magnet as shown in Figs. 1 and 2 is used to show the effectiveness of the proposed IT2FST of OPID. The speed to volt and volt to speed transformation are given in Figs. 11 and 12, respectively. In addition, IT2FST of OPID controller is shown in Fig. 13. The desired speed is equivalent to $2 \mathrm{~V}$ under normal load torque. Three tests of practical implementation are carried out.

Test 1 Uncertainty in the identified SPMSM model under normal load torque with step disturbance speed $(\mathrm{rpm})=2 \mathrm{v}$.

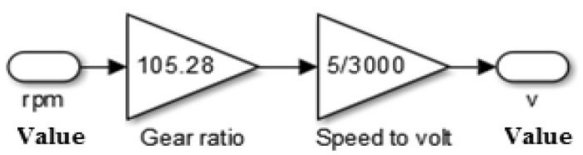

Fig. 11 Block diagram of speed/volt converter

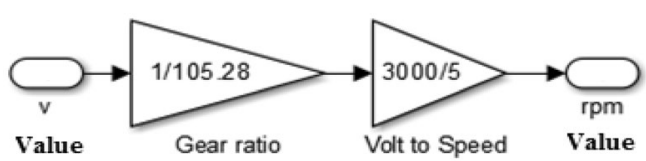

Fig. 12 Block diagram of volt/speed converter 

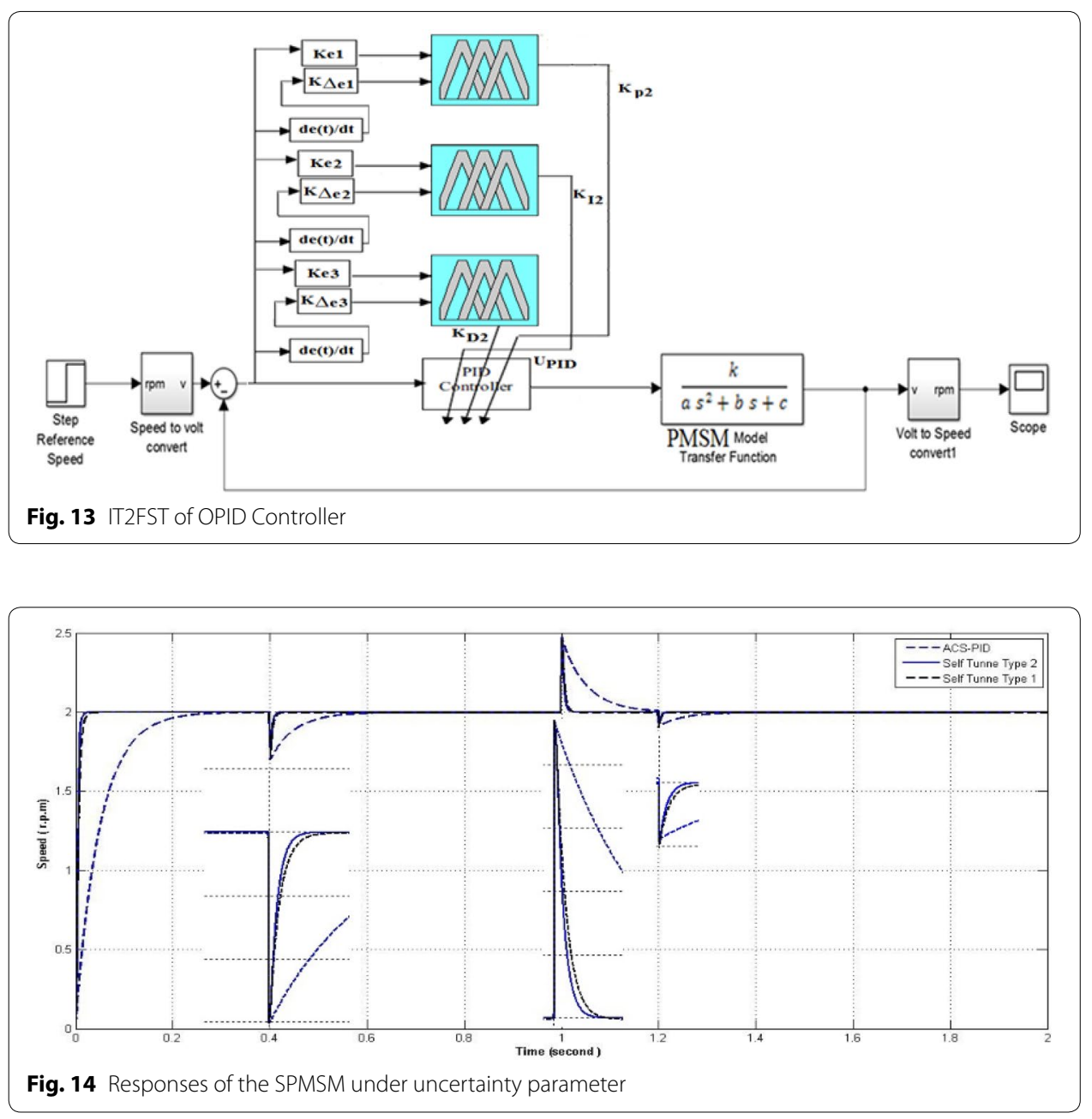

In this situation, a nominal value of $K$ given by Eq. (1) is maintained constant for $0 \leq t \geq 0.4,15 \%$ of $K$ is decreased for $0.4 \leq t \geq 1$ and $5 \%$ of $K$ is increased from nominal value for $1 \leq t \geq 1.2$ and maintained constant for $1.2 \leq t \geq 2$. The system uncertainties are applied to the system driven by each of the three controllers.

The effect of uncertainty in parameter $K$ is illustrated in Fig. 14. It is noted that T1FST of OPID produces better performance in the rise time and the steady-state error, settling time than OPID. Also, it is clear that IT2FST of OPID shows close response to the T1FST of OPID but slightly better. However, OPID shows the worst response (longer rise and settling times).

Test 2 Under load torque and step disturbance speed $(\mathrm{rpm})=2 \mathrm{v}$.

To test the effectiveness of the proposed IT2FST of OPID controller, a sudden increase in the speed by $0.5 v$ at time $(1.5 \mathrm{~s})$ is observed. This leads to a decrease in the load torque. The real practical responses of T1FST of OPID and OPID are compared with their respective simulation results and given in Fig. 15. The time responses of the controller outputs are displayed in Fig. 16. It is clear that the speed time responses have a 


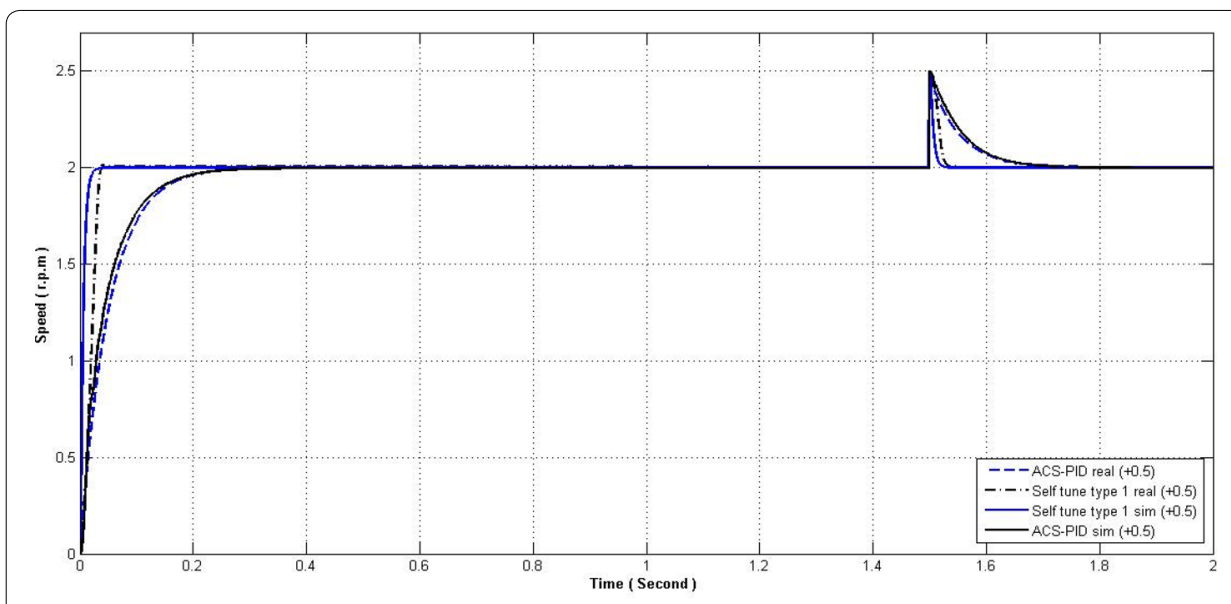

Fig. 15 PMSM system response to T1FST of OPID and OPID

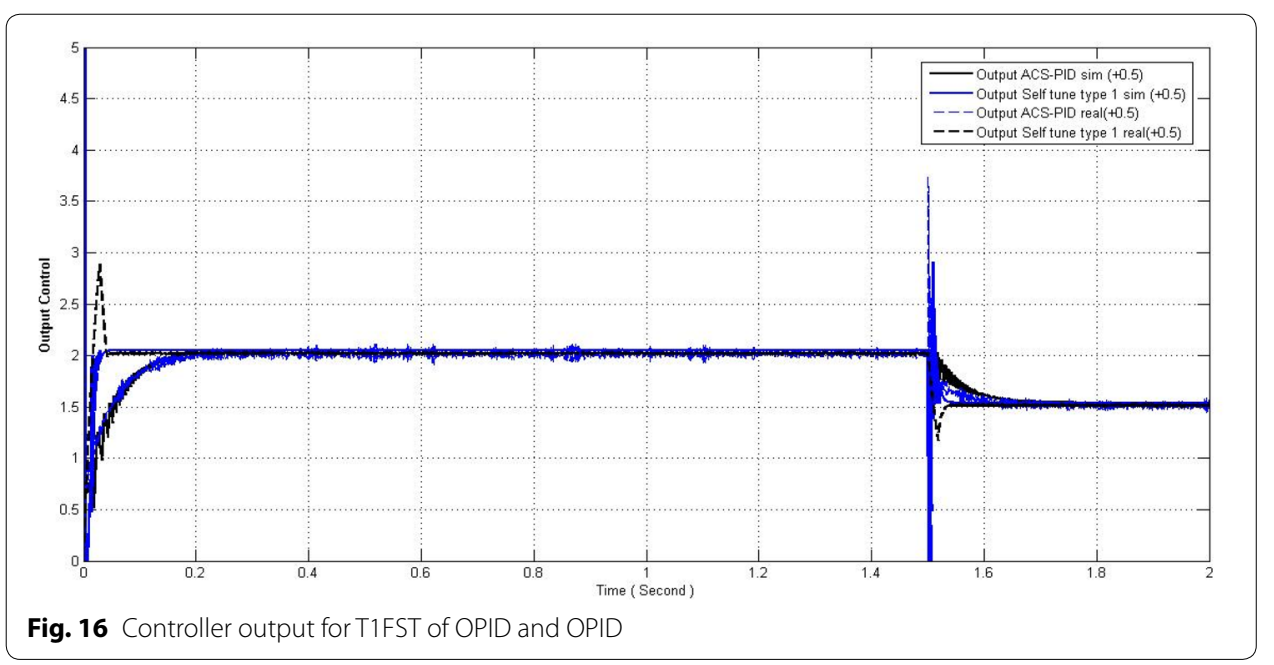

lower overshoot and smaller settling time is seen using T1FST of OPID than the OPID. The controller output of T1FST of OPID for practical simulation has a small overshoot, but it gives better response at this point for speed regulation.

Finally in this test, the controlled system of SPMSM is practical implemented using two types of IT2FST and T1FST of OPID controllers. For each controller as shown in Fig. 17, the practical and simulation responses show close and very near response of IT2FST and T1FST of OPID. In terms with better performance in control, damping the speed response of IT2FST is rapidly driven back to speed desired when compared with the other two controller (T1FST of OPID and OPID) responses. The controller output of IT2FST of OPID for practical simulation has a large value compared to the other controllers, but within permissible range of SPMSM operation as shown in Fig. 18.

Test 3 Over load torque and step disturbance speed $(\mathrm{rpm})=2 \mathrm{v}$. 


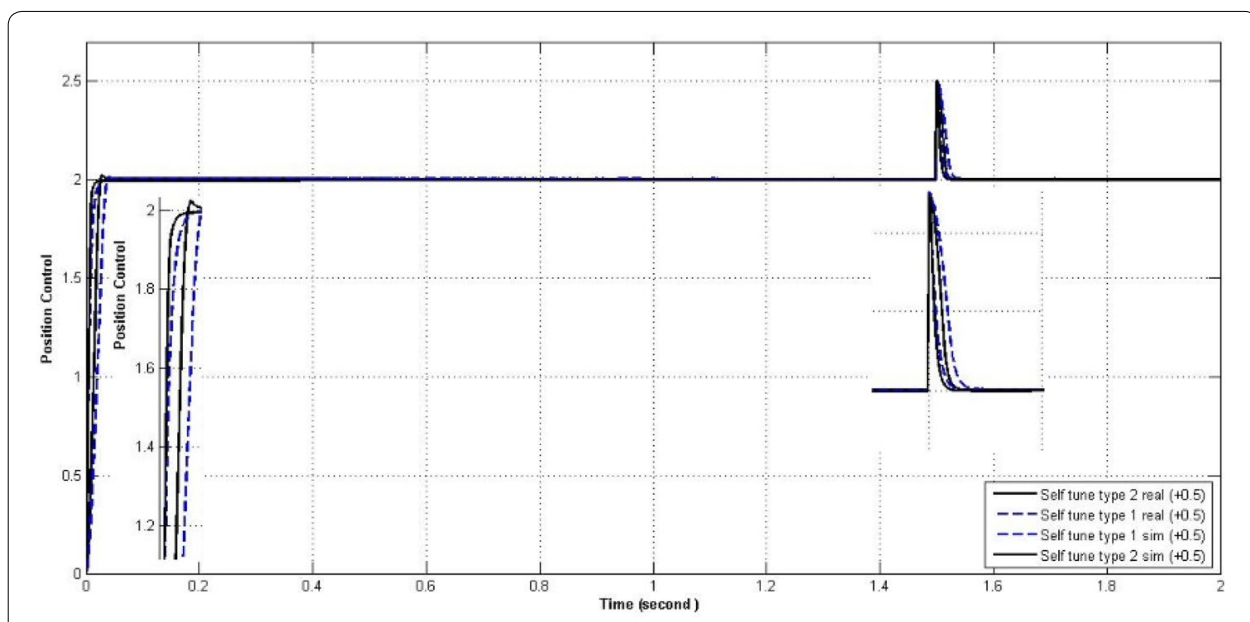

Fig. 17 PMSM system response to T1FST and IT2FST of OPID

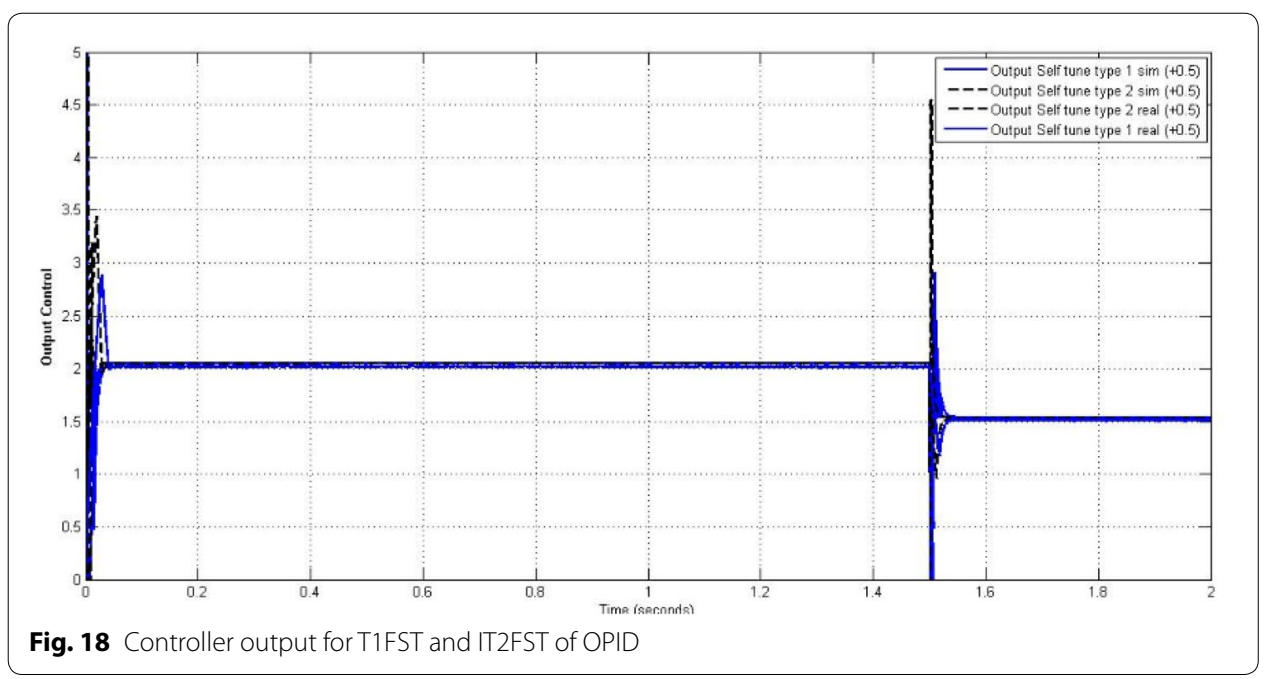

Effectiveness of the proposed IT2FST of OPID controller is verified through a sudden decrease in the speed by $0.5 v$ at time $(1.5 \mathrm{~s})$. This causes increasing the load torque, and consequently, the speed decreases. In this test, firstly, real practical implementation for OPID and T1FS of OPID can be performed and compared to the desired speed. Similarly, T1FS of OPID produces better performance in the rise time and the steady-state error and settling time than OPID as depicted in Fig. 19. The time response of the controller outputs is displayed in Fig. 20. The controller output of IT2FST of OPID for practical simulation has overshoot but the value of the controller output steel with acceptable range gives better response for corresponding speed regulation.

Secondly, real practical implementation for IT2FST and T1FST of OPID controllers is performed. From results in Fig. 21, it is clear that the IT2FST of OPID has better overall performance compared to T1FST of OPID. This improvement is patent in terms of reduction, disturbances and smaller overshoot, which finally yields a much improved 


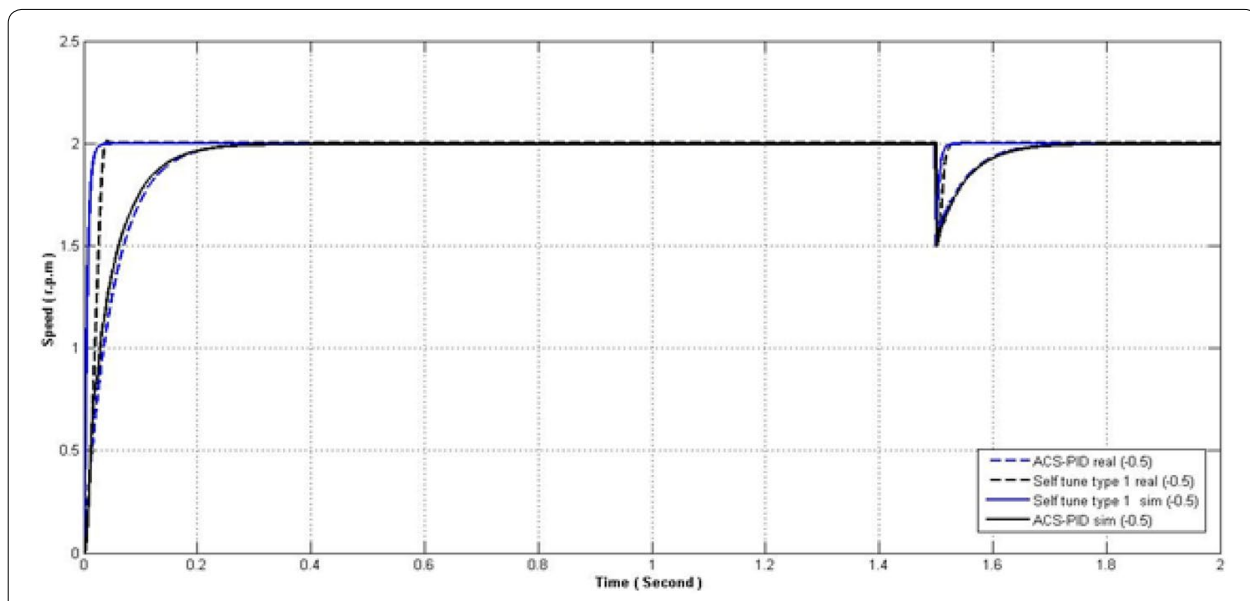

Fig. 19 PMSM system response to T1FST of OPID and OPID

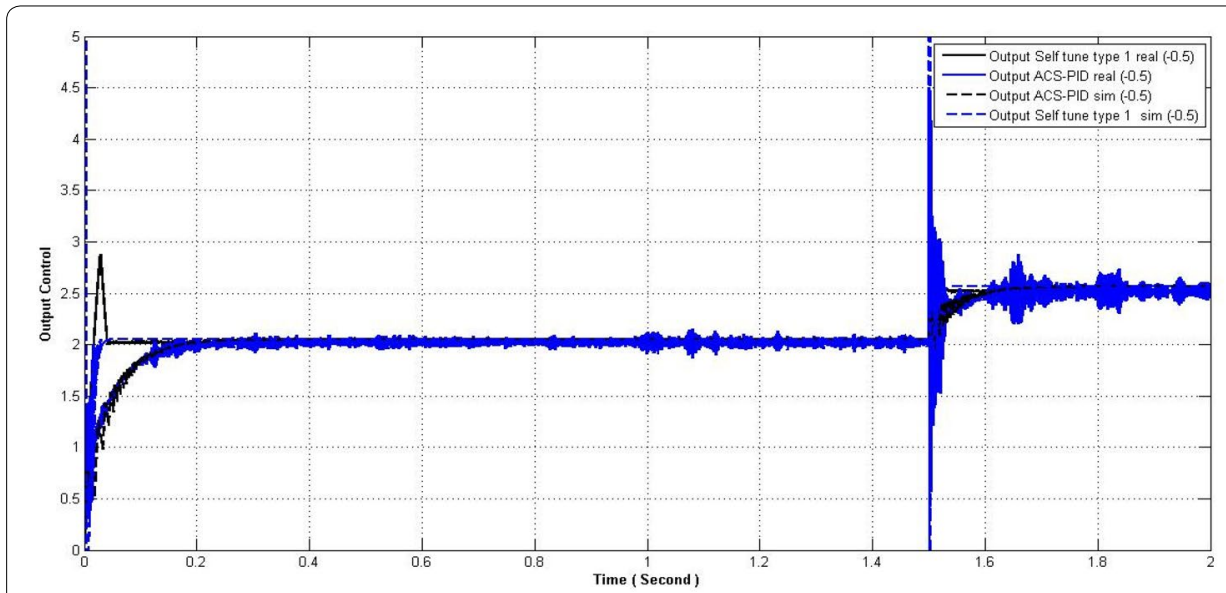

Fig. 20 Controller output for T1FST of OPID and OPID

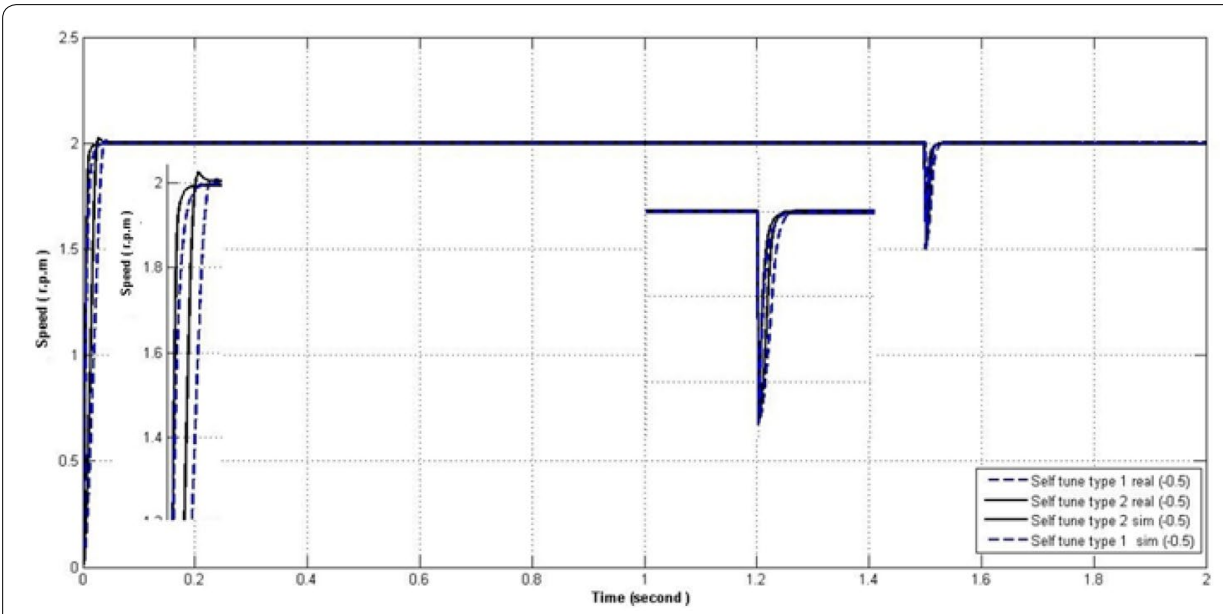

Fig. 21 PMSM system response to T1FST and IT2FST of OPID 


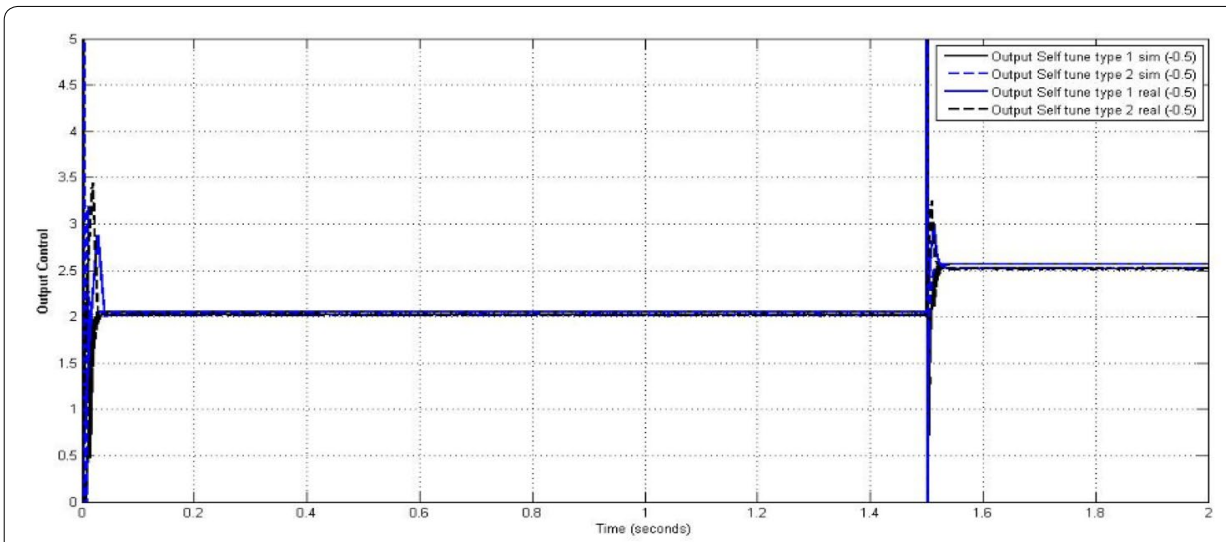

Fig. 22 Controller output for T1FST and IT2FST of OPID

overall performance. The outputs of the controllers are illustrated in Fig. 22, and each one of them is within allowable practical range for operation.

\section{Conclusion}

In this study, a performance comparison between type-1 and type- 2 fuzzy self-tuning of optimal PID is offered. The optimal PID controller is determined using ant colon optimization (ACO). The proposed controllers are used for speed control of industrial SPMSM system. The nonlinear least squares method successfully identified the SPMSM model. The experimental results show that using IT2FST of OPID in real-world applications can be a good option since this type of system is more suitable system to manage levels of uncertainty compared to a conventional type-1 FST controller. The proposed technique is easy to implement the practical design of system controls with external disturbances.

\section{List of symbols}

$\omega(s)$ : Rotor speed; $v(s)$ : Controlled voltage; $k, a, b$ and $c$ : Transfer function parameters; $K_{\mathrm{p}}, K_{\mathrm{i}}$ and $K_{\mathrm{d}}$ : Proportional, integral and differential gains; $K_{\mathrm{ej}}$ : Error input normalizing gain, $i=1,2,3 ; K_{\Delta \mathrm{ei}}$ : $\Delta$ error input normalizing gain, $i=1,2,3$.

\section{Acknowledgements}

I would like to express my deepest appreciation to all those who provided me the possibility to complete this research.

\section{Authors' contributions}

MAAG was involved in design and control implementation; MEB reviewed the simulation results; WMR reviewed the paper; and SS reviewed the paper. All authors read and approved the final manuscript.

\section{Funding}

Research support from author's affiliations.

\section{Availability of data and materials}

The data that support the findings of this study are available from the corresponding author [M. A. Abdel Ghany], upon reasonable request.

\section{Competing interests}

The authors declare that they have no competing interests.

\section{Author details}

${ }^{1}$ Faculty of Engineering, October 6 University, Cairo, Egypt. ${ }^{2}$ Faculty of Engineering - Helwan, University of Helwan, Cairo, Egypt.

Received: 3 September 2019 Accepted: 16 December 2019

Published online: 09 January 2020 


\section{References}

1. Liu H, Li S (2012) Speed control for PMSM servo system using predictive functional control and extended state observer. IEEE Trans Ind Electron 59(2):71-83

2. Seok-Beom L (2006) Closed-loop estimation of permanent magnet synchronous motor parameters by PI controller gain tuning. IEEE Trans Energy Convers 21(4):63-70

3. Zhuang M, Atherton DP (1993) Automatic tuning of optimum PID controllers. IEE Proc D 140(3):216-224

4. Astrom KJ, Hagglund T, Zhang CC, Ho WK (1993) Automatic tuning and adaptation for PID controllers-a survey. IFAC J Control Eng Pract 1(4):699-714

5. Jianguang Z, Zhifeng Z, Renyuan T (2008) IEEE self-tuning PI controller based on neural network for permanent magnet synchronous motor. In: Fourth international conference on natural computation, pp 532-537

6. Chun-Yu D, Gwo-Ruey Y (2007) Optimal PI control of a permanent magnet synchronous motor using particle swarm optimization. In: IEEE ICIC'07 second international conference, pp 255-258

7. Algreer MMF, Kuraz YRM (2008) Design fuzzy self tuning of PID controller for chopper-fed DC motor drive. AlRafidain Eng J 16(2):54-66

8. Abdel Ghany MA, Bahgat ME, Refaey WM, Hassan FN (2017) Design of fuzzy self tuning PID load frequency controller for the Egyptian power system. J Azhar Univ Eng Sect 12(42):77-89

9. El Zoghbyand HM, Abdel Ghany AM (2014) Transient fault ride-through of a multi-generators wind farm using a selftuning fuzzy PID controller. In: The international middle-east power systems conference-MEPCON'2014 Ain Shams University, Cairo, Egypt, December 23-25, 2014

10. Mendel JM (2001) On the importance of interval sets in type-2 fuzzy logic systems. In: Proceedings of the joint 9th IFSA world congress 20th NAFIPS international conference, Vancouver, BC, Canada, 25-28 July, 2001, pp 1647-1652

11. Mendel JM, John RIB (2002) Type-2 fuzzy sets made simple. IEEE Trans Fuzzy Syst 10(2):117-127

12. Mende JM, John RI, Liu F (2006) Interval type-2 fuzzy logic systems made simple. IEEE Trans Fuzzy Syst 14(6):808-821

13. Mendel JM, John RIB (2002) Footprint of uncertainty and its importance to type-2 fuzzy sets. In: Proceeding of 6th IASTED international conference on artificial intelligence and soft computing, Banff, Canada, July 2002, pp 587-592

14. El-Nagar AM, El-Bardini M (2014) Derivation and stability analysis of the analytical structures of the interval type-2 fuzzy PID controller. Appl Soft Comput 24:704-716

15. El-Bardini M, El-Nagar AM (2014) Interval type-2 fuzzy PID controller: analytical structures and stability analysis. Arab J Sci Eng 39:7443-7458

16. Ahmad M. El-Nagar and Mohammad El-Bardini," Practical Implementation for the interval type-2 fuzzy PID controller using a low cost microcontroller" Ain Shams Engineering Journal 2014 5, 475-487

17. Abdel Ghany MA, Abdel Magid H, Abdullah Eissa M, Bahgat ME, Bassuiny A, Sharaf S (2017) Practical implementation for fuzzy self tuning of optimal PID controller to servo permanent magnet synchronous motor. J Al-Azhar Univ Eng Sect 12:1371-1385

18. Boileau T, Leboeuf N, Nahid-Mobarakeh B (2011) Online identification of PMSM parameters: parameter identifiability and estimator comparative study. IEEE Trans Ind Electron 47(4):44-57

19. Wang K, Chiasson J, Bodson M, Tolbert LM (2004) A nonlinear least-squares approach for identification of the induction motor parameters. In: 43rd IEEE conference on decision and control, December 14-17, Atlantis, Paradise Island, Bahamas, pp 3856-3861

20. Abdel Ghany MA, Bahgat ME, Refaey WM, Hassan FN (2014) Ant colony optimum tuning of PID load frequency controller for the egyptian power system. In: Sixteenth international Middle East power systems conference (MEPCON'14), Ain Shams University, Egypt

21. Zeng Q, Tan G (2007) Optimal design of PID controller using modified ant colony system algorithm. In: IEEE, 3rd international conference on natural computation (ICNC)

22. Mendel JM (2007) Type-2 fuzzy sets and systems: an overview. IEEE Comput Intell Mag 2:20-29

23. Mendel JM, Wu H (2007) New results about the centroid of an interval type-2 fuzzy set, including the centroid of a fuzzy granule. Inf Sci 177:360-377

24. Wu H, Mendel JM (2001) Introduction to uncertainty bounds and their use in the design of interval type-2 fuzzy logic systems. In: 2001 IEEE international fuzzy systems conference, pp 662-665

\section{Publisher's Note}

Springer Nature remains neutral with regard to jurisdictional claims in published maps and institutional affiliations. 